\title{
Previsão in silico ADME/T de novos inibidores potenciais contra o vírus da dengue
}

\author{
In silico ADME/T prediction of novel potential inhibitors against dengue virus \\ Predicción in silico ADME/T de nuevos inhibidores potenciales contra el virus del dengue
}

Recebido: 30/03/2021 | Revisado: 09/04/2021 | Aceito: 11/04/2021 | Publicado: 21/04/2021

Jane Stefani da Mata Rodrigues ORCID: https://orcid.org/0000-0002-8003-2488 Faculdade Ciências da Vida, Brasil E-mail: jstefane41@gmail.com

Eduardo Damasceno Costa

ORCID: https://orcid.org/0000-0003-4769-5308

Faculdade Ciências da Vida, Brasil

E-mail: damascenomg13@yahoo.com.br

\begin{abstract}
Resumo
A dengue é uma doença emergente com grande impacto na saúde pública, com milhões de infecções virais ocorrendo anualmente, para a qual ainda não existe uma terapia eficaz. O presente estudo tem como objetivo realizar a predição das propriedades físico-químicas, farmacocinéticas e toxicológicas de candidatos a medicamentos contra dengue. 17 candidatos a fármacos anti-dengue foram desenvolvidos na plataforma PubChem Sketcher V.2.4 ${ }^{\circledR}$. Os parâmetros físico-químicos foram quantificados na plataforma Molinspiration ${ }^{\circledR}$. Posteriormente, os parâmetros farmacocinéticos foram medidos usando a ferramenta SwissADME ${ }^{\circledR}$. Finalmente, a plataforma OSIRIS Property Explorer ${ }^{\circledR}$ foi usada para determinar o efeito toxicológico dos candidatos anti-dengue. Os compostos 8 e 14 não violaram nenhuma das regras instituídas por Lipinski. Todos os outros compostos mostraram mais de uma violação, com os compostos 5,7 e 9-11 apresentando até 3 violações. Quanto à avaliação farmacocinética, dos compostos aqui desenhados apenas os compostos 13 e 14 apresentaram elevada absorção gastrointestinal. Os compostos 2, 15 e 17 têm pelo menos uma pontuação de alto risco para um dos fatores de toxicidade para mutagênese, tumorigênese, efeitos irritantes e reprodutivos. Os compostos 1-4 têm pelo menos uma pontuação de risco intermediária para um dos fatores de toxicidade. Todos os outros compostos têm pontuação de baixo risco para um os fatores de toxicidade. A previsão in silico realizada nesse estudo indicou que os compostos 13 e 14 são as mais promissoras para serem possíveis candidatas anti-dengue e úteis para futuras triagens em testes realizados em células e animais.
\end{abstract}

Palavras-chave: Dengue; Denv; In silico; Ferramentas computacionais; Modelagem molecular.

\begin{abstract}
Dengue is an emerging disease with a major impact on public health, with millions of viral infections occurring annually, for which there is still no effective therapy. The present study aims to predict the physicochemical, pharmacokinetic and toxicological properties of candidates for drugs against dengue. 17 candidates for anti-dengue drugs were developed on the PubChem Sketcher V. $2.4^{\circledR}$ platform. The physical-chemical parameters were quantified on the Molinspiration ${ }^{\circledR}$ platform. Subsequently, the pharmacokinetic parameters were measured using the SwissADME ${ }^{\circledR}$ tool. Finally, the OSIRIS Property Explorer ${ }^{\circledR}$ platform was used to determine the toxicological effect of anti-dengue candidates. Compounds 8 and 14 did not violate any of the rules instituted by Lipinski. All other compounds showed more than one violation, with compounds 5, 7 and 9-11 showing up to 3 violations. As for the pharmacokinetic evaluation, of the compounds designed here only compounds 13 and 14 showed high gastrointestinal absorption. Compounds 2, 15 and 17 have at least a high-risk score for one of the toxicity factors for mutagenesis, tumorigenesis, irritating and reproductive effects. Compounds 1-4 have at least an intermediate risk score for one of the toxicity factors. All other compounds have low risk scores for one of the toxicity factors. The in silico prediction made in that study indicated that compounds 13 and 14 are the most promising for being possible anti-dengue candidates and useful for future screening in tests performed on cells and animals.
\end{abstract}

Keywords: Dengue; Denv; In silico; Computational tools; Molecular modeling.

\section{Resumen}

El dengue es una enfermedad emergente con un gran impacto en la salud pública, con millones de infecciones virales que ocurren anualmente, para las cuales aún no existe una terapia efectiva. Este estudio tiene como objetivo predecir las propiedades fisicoquímicas, farmacocinéticas y toxicológicas de candidatos a fármacos contra el dengue. Se desarrollaron 17 candidatos para medicamentos contra el dengue en la plataforma PubChem Sketcher V.2.4 ${ }^{\circledR}$. Los parámetros físico-químicos se cuantificaron en la plataforma Molinspiration ${ }^{\circledR}$. Posteriormente, se midieron los parámetros farmacocinéticos utilizando la herramienta SwissADME ${ }^{\circledR}$. Finalmente, se utilizó la plataforma OSIRIS 
Property Explorer ${ }^{\circledR}$ para determinar el efecto toxicológico de los candidatos anti-dengue. Los compuestos 8 y 14 no violaron ninguna de las reglas instituidas por Lipinski. Todos los demás compuestos mostraron más de una violación, y los compuestos 5, 7 y 9-11 mostraron hasta 3 violaciones. En cuanto a la evaluación farmacocinética, de los compuestos aquí diseñados, solo los compuestos 13 y 14 mostraron una alta absorción gastrointestinal. Los compuestos 2, 15 y 17 tienen al menos una puntuación de riesgo alta para uno de los factores de toxicidad para mutagénesis, tumorigénesis, efectos irritantes y reproductivos. Los compuestos 1-4 tienen al menos una puntuación de riesgo intermedia para uno de los factores de toxicidad. Todos los demás compuestos tienen una puntuación de riesgo baja para uno de los factores de toxicidad. La predicción in silico realizada en este estudio indicó que los compuestos 13 y 14 son los más prometedores por ser posibles candidatos anti-dengue y útiles para futuros cribados en pruebas realizadas en células y animales.

Palabras clave: Dengue; Denv; In silico; Herramientas computacionales; Modelado molecular.

\section{Introduçãa}

A dengue é uma arbovirose transmitida através da picada da fêmea do mosquito Aedes aegypti. Trata-se de uma doença febril aguda, dinâmica e sistêmica, de notificação compulsória bastante prevalente em todo o mundo (Brady \& Hay, 2020). Os dados epidemiológicos mais recentes indicam que aproximadamente 100 milhões de infecções pelo vírus da dengue tem ocorrido anualmente em todo o mundo. Além disso, estima-se que aproximadamente 3,9 bilhões de pessoas distribuídas em 128 países vivem com o risco de se infectar por essa doença (Pollett et al., 2018).

A forma clássica e a hemorrágica da dengue são causadas por vírus do gênero Flavivírus (Murugesan \& Manoharan, 2019). O vírus da dengue possui genomas compostos de RNA de cadeia simples com pouca capacidade de codificação, distintos por 4 sorotipos virais (DENV-1, DENV-2, DENV-3 e DENV-4) (Waman et al., 2016). Um quinto sorotipo, o DENV5 foi descoberto em 2013, em amostras de macacos da região da Malásia, mas que também pode circular entre seres humanos (Powers \& Setzer, 2016).

Em 2015 foi desenvolvida pelo laboratório farmacêutico Sanofi Pasteur a primeira vacina tetravalente contra o vírus da dengue, a Dengvaxia ${ }^{\circledR}$. Apesar da sua relativa eficiência terapêutica, dados mostram que essa vacina poderia levar a um maior risco de hospitalização em indivíduos não infectados e também causar casos mais graves da doença em indivíduos soronegativos. A Dengvaxia ${ }^{\circledR}$ está atualmente restrita para uso em situação de endemias (Guy et al., 2017). Além da vacina, potenciais medicamentos anti-dengue também vêm sendo avaliados em ensaios clínicos. As principais moléculas que já foram ensaiadas são a cloroquina, a celgosivir, o balapiravir, a prednisona, a lovastatina, a ivermectina e a ribavirina. No entanto, nenhuma dessas moléculas foram aprovadas para tratamento contra a dengue (Beesetti et al., 2016). Diante desse cenário, pesquisas realizadas pelas grandes indústrias farmacêuticas que envolvam a identificação de novos candidatos a fármacos para o tratamento da dengue permanecem ainda como um grande desafio.

Nos últimos tempos intensificaram propostas que consideram importante a redução no uso de animais para processo de experimentação laboratorial que esteja relacionado a descoberta de novas entidades químicas com valor terapêutico. Uma das principais abordagens desenvolvidas em substituição ao uso de animais são os estudos que utilizam ferramentas computacionais ou softwares de pesquisa, os chamados modelos in silico (Madden et al., 2020).

Os modelos in silico são métodos computacionais que fazem uso de algoritmos matemáticos e que visam a otimização de moléculas com maior potencial de desenvolvimento. Os modernos sistemas computacionais têm permitido a avaliação de propriedades físico-químicas importantes para a ação de fármacos (peso da molécula, lipofilicidade, aceptores e doadores de hidrogênio) e de características farmacocinéticas: absorção, distribuição, metabolismo, excreção e toxicidade (ADME-Tox). Cabe ressaltar que os modelos computacionais são ferramentas extremamente úteis para o processo de descoberta de novas fármacos, mas ainda assim é fundamental a integração destes modelos com estudos in vitro (em células), in vivo (modelos animais) e com os ensaios clínicos (humanos) no intuito de maximizar as chances de sucesso no desenvolvimento de um novo medicamento (Vavougios et al., 2018). Os programas que avaliam parâmetros farmacocinéticos in silico emergiram 
recentemente como importante ferramenta para a descoberta de novos fármacos. Os softwares de modelagem molecular se baseiam em métodos matemáticos complexos e robustos que permitem avaliar a cinética e a predição de alguns efeitos adversos de um candidato a fármaco. É bastante comum que durante o processo de descoberta e desenvolvimento de fármacos pela indústria farmacêutica diversas moléculas potencialmente ativas possam ser descartadas devido a problemas relacionados a características farmacocinéticas e a problemas de toxicidade (Ferreira \& Andricopulo, 2019). Diante disto, a utilização dos métodos in silico, tornou-se uma ferramenta fundamental permitindo a redução de custos e de tempo em relação aos modelos animais, além da capacidade de processamento de grande quantidade de dados a respeito de uma quantidade muito grande de moléculas potencialmente ativas (Rao \& Srinivas, 2011). Diante do cenário apresentado, essa pesquisa tem como objetivo geral realizar a previsão de propriedades físico-químicas, farmacocinéticas e toxicológicas de dezessete candidatos a fármacos contra o vírus da dengue utilizando ferramentas in silico.

\section{Metodologia}

Para garantir a relevância, a confiabilidade e a validade do processo de investigação científica é fundamental a adesão de um método de pesquisa preciso que atenda às necessidades do problema investigado (Vera, 1989). O processo científico, portanto, pode ser categorizado quanto à sua finalidade, objetivo, abordagem, método e procedimento metodológico (Gil, 2010; Vergara, 2006). Em virtude disso, quanto a sua finalidade, trata-se de uma pesquisa explicativa, baseada em métodos experimentais, através da apresentação de um problema e da elucidação de hipóteses especulativas que tentarão solucioná-lo (Vergara, 2006). Desta maneira, quanto aos objetivos, caracteriza-se como descritiva e explicativa, tendo intensão de avaliar, descrever e elucidar o desenho e a previsão das principais propriedades físico-químicas, farmacocinéticas e toxicológicas dos análogos anti-dengue, através de estudos in silico em modelos de modelagem molecular. A pesquisa contará com uma abordagem baseada em processos quantitativos, na qual os perfis de absorção, distribuição, metabolização, eliminação e de toxicidade dos análogos analisados serão passíveis de serem medidos numericamente (Rosental, Claude; Frémontier-Murphy, 2001). Por fim, quanto aos procedimentos experimentais, essa é uma pesquisa do tipo experimental, cuja abordagem metodológica apresentará variáveis que serão controladas (Lakatos, Eva Maria, Marconi; 2011).

Inicialmente nós realizamos o desenho racional dos 17 candidatos a fármacos contra a dengue na plataforma PubChem Sketcher V. $2.4^{\circledast}$ (https://pubchem.ncbi.nlm.nih.gov/edit3/index.html). Trata-se de uma ferramenta online para desenho de estruturas químicas, incluindo as orgânicas, polímeros e compostos organometálicos. Avaliamos os parâmetros físico-químicos e os quantificamos através da ferramenta Molinspiration ${ }^{\circledR}$ (http://www.molinspiration.com). Com uso dessa ferramenta é possível realizar o cálculo, peso molecular, do LogP, número de átomos aceptores de ligação de hidrogênio, número de átomos doadores de ligação de hidrogênio. Posteriormente, os parâmetros de absorção, distribuição, metabolismo e excreção foram medidos usando a ferramenta SwissADME ${ }^{\circledR}$ (http://www.swissadme.ch). Trata-se de uma plataforma online gratuita que estima parâmetros farmacocinéticos. Por fim, foi utilizada a plataforma OSIRIS Property Explorer ${ }^{\circledR}$ (http://www.organicchemistry.org/prog/peo) para determinar o efeito toxicológico dos candidatos a fármacos. O OSIRIS permite presumir o potencial irritante, mutagênico, reprodutivo e cancerígeno dos compostos. Ressaltamos que todas as plataformas aqui elencadas são devidamente validadas e amplamente utilizadas em outros estudos científicos (Thangarasu et al., 2018).

\section{Resultados}

A Figura 1 apresenta o desenho de 17 estruturas químicas construídas na plataforma online PubChem Sketcher ${ }^{\circledR}$. Todas essas moléculas foram desenhadas como possíveis candidatos à fármacos usados contra o vírus da dengue. As 
Research, Society and Development, v. 10, n. 4, e53010414459, 2021

(CC BY 4.0) | ISSN 2525-3409 | DOI: http://dx.doi.org/10.33448/rsd-v10i4.14459

modificações nos análogos foram propostas racionalmente baseadas em dados de relação estrutura atividade (REA) disponíveis em (Dighe et al., 2019).

Figura 1: Desenho racional de 17 estruturas químicas com possível ação anti-dengue.

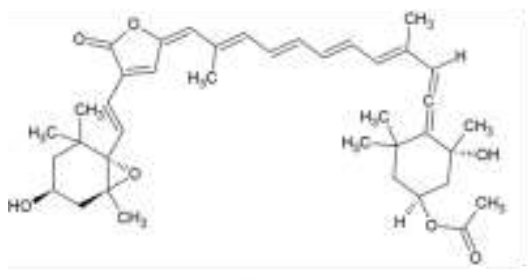

Peridinina

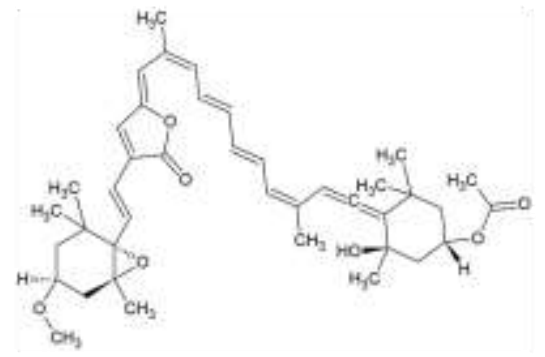

Composto 3

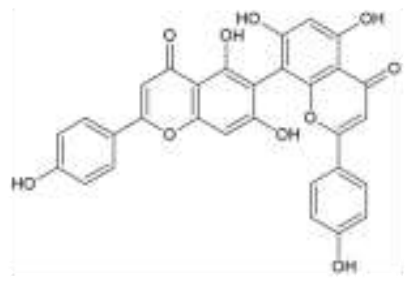

Agatisflavona

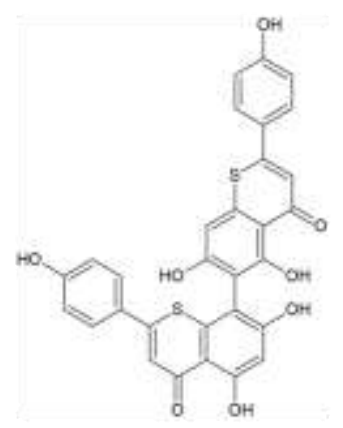

Composto 7

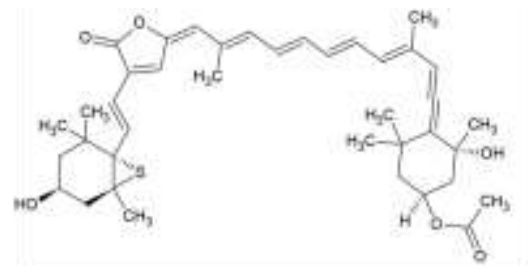

Composto 2

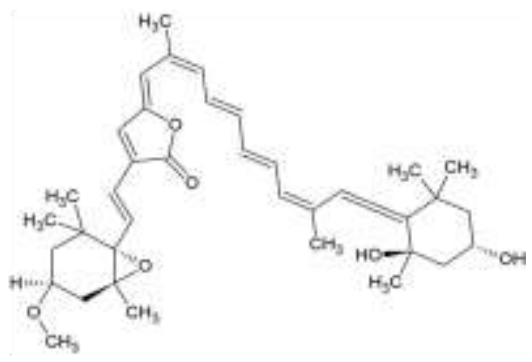

Composto 4

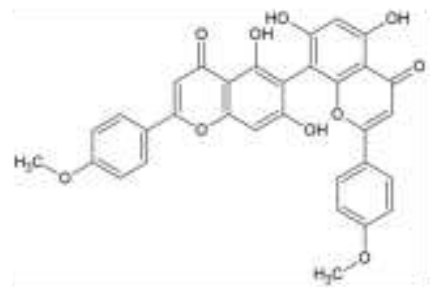

Composto 6

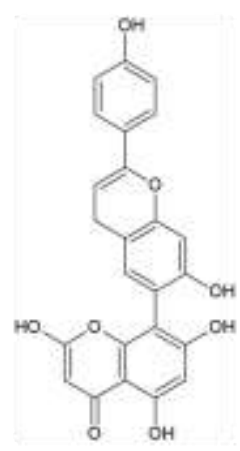

Composto 8 

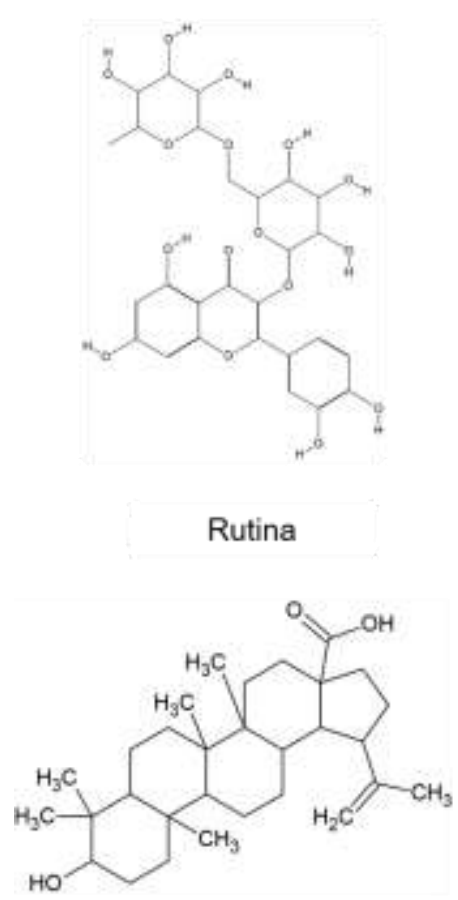

Ácido betulínico

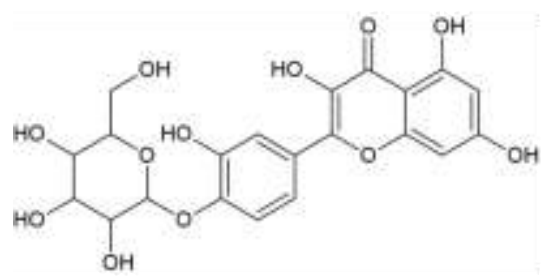

Composto 15

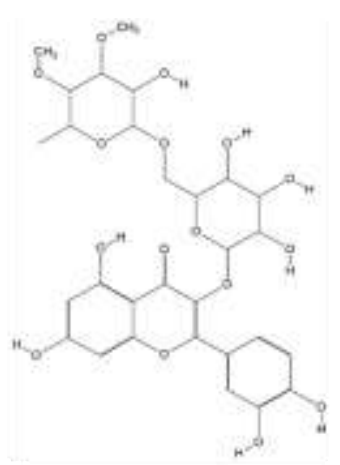

Composto 10

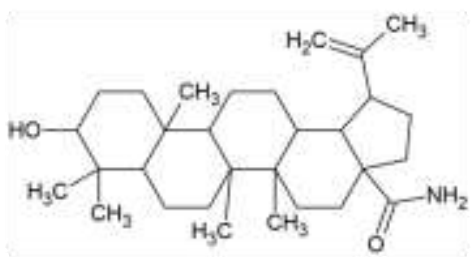

Composto 13

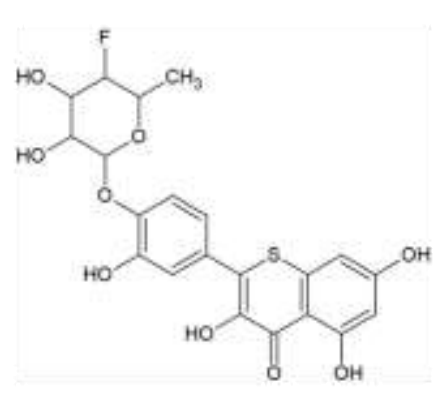

Composto 16

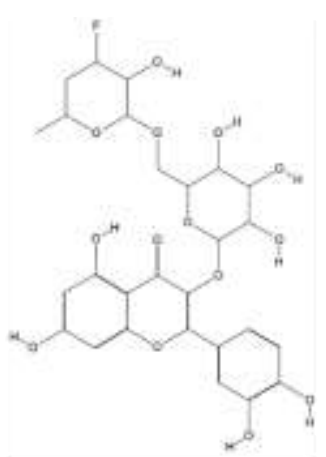

Composto 11

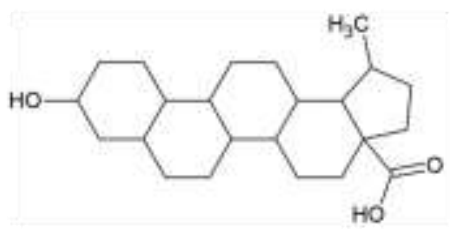

Composto 14

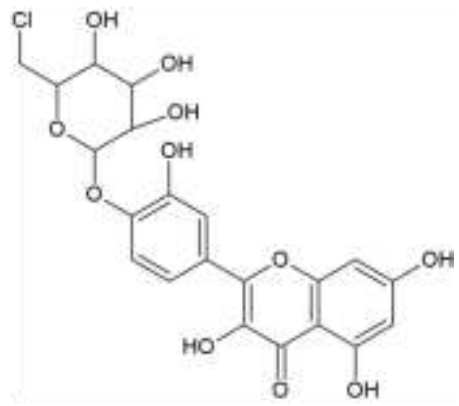

Composto 17

Fonte: Autores (2020).

O Quadro 1 mostra dados físico-químicos das 17 estruturas químicas com ação anti-dengue. No quadro estão dispostas informações a respeito do volume molecular, coeficiente de partição octanol/água (LogP), área de superfície polar (PSA), massa molecular, número de átomos doadores de ligação de hidrogênio (nON), número de ligações rotativas (nrotb), número de átomos aceptores de ligação de hidrogênio (nOHNH), além do possível número de violações a regra de Lipinski. Os valores aqui obtidos foram obtidos usando a plataforma Molinspiration ${ }^{\circledR}$. Os compostos 8 e 14 não violaram nenhum dos pontos da regra de Lipinski. Os compostos 5, 7, 9, 10 e 11 foram os compostos que violaram a regra de Lipinski em pelo menos 3 propriedades. 
Quadro 1. Propriedades físico-químicas de 17 estruturas químicas anti-dengue.

\begin{tabular}{|c|c|c|c|c|c|c|c|c|}
\hline Composto & Volume & $\mathbf{L o g P}$ & $\begin{array}{c}\text { Área de } \\
\text { superfície } \\
\text { polar } \\
\mathbf{( P S A )} \\
\mathbf{( \mathbf { A } )}\end{array}$ & $\begin{array}{c}\text { Massa } \\
\text { molecular } \\
\mathbf{( g / m o L )}\end{array}$ & $\mathbf{n O N}$ & nrotb & nOHNH & $\begin{array}{c}\text { Violações } \\
\text { a regra de } \\
\text { Lipinski }\end{array}$ \\
\hline $\mathbf{1}$ & 613.06 & 7.24 & 109.50 & 630.82 & 7 & 9 & 2 & 2 \\
\hline $\mathbf{2}$ & 622.20 & 7.78 & 96.97 & 646.89 & 6 & 9 & 2 & 2 \\
\hline $\mathbf{3}$ & 630.59 & 7.86 & 98.51 & 644.85 & 7 & 10 & 1 & 2 \\
\hline $\mathbf{4}$ & 594.08 & 7.15 & 92.43 & 602.81 & 6 & 8 & 2 & 2 \\
\hline $\mathbf{5}$ & 435.46 & 5.16 & 181.79 & 538.46 & 10 & 3 & 6 & 3 \\
\hline $\mathbf{6}$ & 470.52 & 6.23 & 159.80 & 566.52 & 10 & 5 & 4 & 2 \\
\hline $\mathbf{7}$ & 453.75 & 6.44 & 155.51 & 570.60 & 8 & 3 & 6 & 3 \\
\hline $\mathbf{8}$ & 353.85 & 4.01 & 140.59 & 432.38 & 8 & 2 & 5 & 0 \\
\hline $\mathbf{9}$ & 496.07 & -1.06 & 269.43 & 610.52 & 16 & 6 & 10 & 3 \\
\hline $\mathbf{1 0}$ & 531.12 & -0.27 & 247.44 & 638.58 & 16 & 8 & 8 & 3 \\
\hline $\mathbf{1 1}$ & 484.94 & 0.35 & 228.97 & 596.51 & 14 & 6 & 8 & 3 \\
\hline $\mathbf{1 2}$ & 472.04 & 7.04 & 57.53 & 456.71 & 3 & 2 & 2 & 1 \\
\hline $\mathbf{1 3}$ & 475.31 & 6.53 & 63.32 & 455.73 & 3 & 2 & 3 & 1 \\
\hline $\mathbf{1 4}$ & 362.78 & 4.61 & 57.53 & 360.54 & 3 & 1 & 2 & 0 \\
\hline $\mathbf{1 5}$ & 372.21 & -0.33 & 210.50 & 464.38 & 12 & 4 & 8 & 2 \\
\hline $\mathbf{1 6}$ & 370.00 & 1.81 & 156.91 & 466.44 & 9 & 3 & 6 & 1 \\
\hline $\mathbf{1 7}$ & 377.72 & 0.91 & 190.28 & 482.82 & 11 & 4 & 7 & 2 \\
\hline
\end{tabular}

Fonte: Autores (2020).

O Quadro 2 indica os resultados relacionados ao perfil farmacocinético dos 17 análogos anti-dengue. Os dados farmacocinéticos foram obtidos na plataforma SwissADME ${ }^{\circledR}$ e foram elencadas informações sobre a absorção gastrointestinal (AGi), a penetração na barreira hematoencefálica (BHE) e dados sobre a inibição das enzimas do citocromo P450 (CYP3A4 e CYP2D6). Adicionalmente, foi verificado a acessibilidade sintética (AS) de cada uma das estruturas químicas. Entre os análogos aqui desenhados os compostos 13 e 14 apresentaram alta absorção por via gastrintestinal. Todos os outros 15 compostos propostos mostraram baixa absorção pelo trato gastrintestinal. 
Quadro 2. Dados das principais propriedades farmacocinéticas de 17 análogos anti-dengue.

\begin{tabular}{|c|c|c|c|c|c|}
\hline Compostos & AGi & BHE & Inibe CYP3A4 & Inibe CYP2D6 & AS \\
\hline $\mathbf{1}$ & Baixa & Não & Não & Não & 7.51 \\
\hline $\mathbf{2}$ & Baixa & Não & Não & Não & 7.56 \\
\hline $\mathbf{3}$ & Baixa & Não & Não & Não & 7.61 \\
\hline $\mathbf{4}$ & Baixa & Não & Não & Não & 7.46 \\
\hline $\mathbf{5}$ & Baixa & Não & Não & Não & 4.17 \\
\hline $\mathbf{6}$ & Baixa & Não & Não & Não & 4.33 \\
\hline $\mathbf{7}$ & Baixa & Não & Não & Não & 3.92 \\
\hline $\mathbf{8}$ & Baixa & Não & Não & Não & 3.93 \\
\hline $\mathbf{9}$ & Baixa & Não & Não & Não & 6.52 \\
\hline $\mathbf{1 0}$ & Baixa & Não & Não & Não & 6.75 \\
\hline $\mathbf{1 1}$ & Baixa & Não & Não & Não & 6.38 \\
\hline $\mathbf{1 2}$ & Baixa & Não & Não & Não & 5.63 \\
\hline $\mathbf{1 3}$ & Alta & Não & Não & Não & 5.41 \\
\hline $\mathbf{1 4}$ & Alta & Sim & Não & Não & 4.53 \\
\hline $\mathbf{1 5}$ & Baixa & Não & Não & Não & 5.23 \\
\hline $\mathbf{1 6}$ & Baixa & Não & Sim & Não & 5.05 \\
\hline $\mathbf{1 7}$ & Baixa & Não & Sim & Não & 5.27 \\
\hline
\end{tabular}

Fonte: Autores (2020).

O Quadro 3 apresenta os resultados toxicológicos para os 17 análogos anti-dengue alcançados usando a plataforma OSIRIS Property Explorer ${ }^{\circledR}$. As informações adquiridas nesse sistema online envolvem a chance potencial das estruturas químicas aqui propostas em causar mutagênese, tumorogênese, efeitos irritantes e efeitos no sistema reprodutor. Os análogos anti-dengue 2, 15 e 17 possuem pelo menos um score de alto risco para um dos fatores de toxicidade investigado. Os compostos 1,2,3 e 4 apresentam pelo menos um score de risco intermediário para um dos fatores de toxicidade. Todos os outros compostos apresentam score de risco baixo para mutagênese, tumorogênese, efeitos irritantes e reprodutivos.

Quadro 3. Dados toxicológicos de 17 análogos anti-dengue.

\begin{tabular}{|c|c|c|c|c|}
\hline Composto & Mutagênese & Tumorogênese & Efeitos irritante & $\begin{array}{c}\text { Efeitos } \\
\text { Reprodutivos }\end{array}$ \\
\hline 1 & Risco intermediário & Baixo risco & Baixo risco & Baixo risco \\
\hline 2 & Risco intermediário & Baixo risco & Baixo risco & Alto risco \\
\hline 3 & Risco intermediário & Baixo risco & Baixo risco & Baixo risco \\
\hline 4 & Risco intermediário & Baixo risco & Baixo risco & Baixo risco \\
\hline 5 & Baixo Risco & Baixo Risco & Baixo Risco & Baixo Risco \\
\hline 6 & Baixo Risco & Baixo Risco & Baixo Risco & Baixo Risco \\
\hline 7 & Baixo Risco & Baixo Risco & Baixo Risco & Baixo Risco \\
\hline 8 & Baixo Risco & Baixo Risco & Baixo Risco & Baixo Risco \\
\hline 9 & Baixo Risco & Baixo Risco & Baixo Risco & Baixo Risco \\
\hline 10 & Baixo Risco & Baixo Risco & Baixo Risco & Baixo Risco \\
\hline 11 & Baixo Risco & Baixo Risco & Baixo Risco & Baixo Risco \\
\hline 12 & Baixo Risco & Baixo Risco & Baixo Risco & Baixo Risco \\
\hline 13 & Baixo Risco & Baixo Risco & Baixo Risco & Baixo Risco \\
\hline 14 & Baixo Risco & Baixo Risco & Baixo Risco & Baixo Risco \\
\hline 15 & Alto Risco & Baixo Risco & Baixo Risco & Baixo Risco \\
\hline 16 & Baixo Risco & Baixo Risco & Baixo Risco & Baixo Risco \\
\hline 17 & Alto Risco & Alto Risco & Alto Risco & Alto Risco \\
\hline
\end{tabular}

Fonte: Autores (2020). 


\section{Discussão}

Os métodos de modelagem molecular são largamente utilizados em pesquisas que envolvam a descoberta e o desenvolvimento de fármacos. O desenho, a análise de características físico-químicas, de propriedades farmacocinéticas, farmacodinâmicas e toxicológicas in silico deu origem a novas moléculas que se encontram em ensaios clínicos robustos. Consequentemente, considerando a importância dos estudos in silico no processo de descoberta de fármacos, este estudo foi conduzido com intuito de caracterizar potenciais candidatos a fármacos anti-dengue.

Estudos que avaliam propriedades farmacocinéticas (ADME-Tox) de novas entidades químicas com possível valor terapêutico tem disso amplamente publicados nos últimos anos (Hage-Melim et al., 2020). Adicionalmente, um estudo recente identificou por meio de analises computacionais potenciais inibidores da NS-3 Helicase que podem ser úteis no também para o tratamento da Dengue (Halim et al., 2017). Sabendo da importância dos estudos de modelagem molecular, essa pesquisa avaliou inicialmente as propriedades físico-químicas dos 17 candidatos a fármacos anti-dengue.

A regra de 5 de Lipinski é frequentemente usada em estudos dessa natureza para prever a biodisponibilidade de potenciais moléculas com propriedades farmacoterapêuticas. De acordo com essa regra um candidato a fármaco será ativo por administração oral se: 1) o peso molecular for inferior a 500 u.m.a, 2) o coeficiente de partição octanol / água calculado (Log P) for menor que 5,3) houver menos de 5 doadores de ligação de hidrogênio (grupos $\mathrm{OH}$ e $\mathrm{NH}$, por exemplo) e, 4) existir menos de dez aceptores de ligação de hidrogênio (notavelmente $\mathrm{N}$ e O) (Lipinski et al., 1997). As propriedades dos derivados anti-dengue foram calculadas usando a plataforma Molinspiration. Os compostos 8 e 14 não violaram nenhuma das regras instituídas por Lipinski. Todos os outros compostos mostraram mais de uma violação, sendo que os compostos 5, 7, 9, 10 e 11 apresentaram até 3 violações a regra dos cinco.

A lipofilia ou a hidrofilia de um composto químico é indicada pelo $\operatorname{LogP}$ (coeficiente octanol/água). Os valores de LogP dos compostos aqui desenhados (1-7) e (12-14) mostram-se maior que 5 e estão em clara violação a regra de Lipinski. O peso molecular das estruturas (1-7) e (9-11) também se mostraram acima do valor de 500 u.m.a. Moléculas grandes geralmente tem dificuldade em atravessar barreiras biológicas presentes no organismo humano. $\mathrm{O}$ conjunto de dados obtidos desses dois tópicos anteriores sugerem que os compostos listados provavelmente apresentam baixa permeabilidade através de membranas biológicas.

O número de aceptores de ligações de hidrogênio foram violados nos compostos (9-11), 15 e 17. O número de doadores de ligações de hidrogênio foi violado nos compostos 5, 7, (9-11) e (15-17).

A área de superfície polar (TPSA) e número de ligações rotacionais de fármacos também são bons indicadores de uma boa biodisponibilidade oral. O TPSA está correlacionado com o número de ligações de hidrogênio de uma molécula. E os valores da maioria dos medicamentos comerciais com boa biodisponibilidade oral apresentam TPSA menor que 140 Å. Para os candidatos a fármacos anti-dengue propostos aqui os compostos (5-11) e (15-17) estão bem acima do parâmetro limite para TPSA.

No mesmo sentido, estudos indicam que para uma biodisponibilidade favorável por via oral o número de ligações rotacionais deve ser menor ou igual a 10. Nesse estudo, nenhum candidato a fármaco violou essa regra.

Posteriormente, foram previstos nesse trabalho os dados sobre a farmacocinética dos 17 candidatos a fármacos antidengue. Dos compostos aqui desenhados apenas os compostos 13 e 14 apresentaram alta absorção por via gastrintestinal. Todos os outros 15 compostos mostram baixa absorção pelo trato gastrintestinal (TGI). A boa absorção no TGI é um importante parâmetro na comodidade posológica de um candidato a fármaco. Os parâmetros farmacocinéticos também revelaram que apenas o composto 14 é capaz de atravessar a barreira hematoencefálica.

As análises dos dados do metabolismo dos candidatos a fármacos anti-dengue mostram que nenhum dos compostos inibem a isoenzima CYP2D6 e apenas os compostos 16 e 17 inibem a enzima CYP3A4. O metabolismo de fármacos envolve 
um conjunto de reações de biotransformação que geralmente modificam essas moléculas em entidades químicas mais polares para que sejam eliminados na urina. A inibição das isoenzima pertencentes ao sistema CYP450 é uma das principais causas de interações medicamentosas, efeitos adversos ou tóxicos devido à menor purificação e acúmulo do fármaco ou de seus metabólitos no organismo (Kirchmair et al., 2015). Por fim o quadro 2 mostra os dados a respeito da acessibilidade sintética (AS). Os parâmetros de AS variam entre 1 (moléculas facilmente sintetizadas) e 10 (moléculas dificilmente sintetizadas) (Daina et al., 2017). Nos dados aqui apresentados observamos que os compostos anti-dengue com maior dificuldade de síntese são os (1-4).

Os resultados de risco de toxicidade dos candidatos a fármacos desenhados aqui foram previstos no programa Osiris. Este programa online faz previsão com base na similaridade dos grupos funcionais presentes no composto investigado e os compara com dados extensivos de outros compostos testados em experimentos in vitro e in vivo e armazenados num banco de dados do programa. Os resultados da triagem virtual são avaliados e codificados em um escore que foi especificado em baixo, intermediário e alto risco de toxicidade para os fatores: mutagênese, tumorogênese, efeitos irritantes e reprodutivos. Os compostos 2, 15 e 17 apresentam pelo menos um score de alto risco para um dos fatores de toxicidade. Já os compostos (1-4) apresentam pelo menos um score de risco intermediário para um dos fatores de toxicidade. Todos os outros compostos apresentam score de risco baixo para mutagênese, tumorogênese, efeitos irritantes e reprodutivos. Analisando pontualmente as estruturas 13 e 14 que foram as duas únicas que mostram alta absorção via TGI pode-se afirmar ainda que ambas apresentaram baixo risco para os fatores analisados.

Como pudemos verificar no estudo realizado, a estratégia de modelagem molecular aborda diferentes modelos preditivos a fim de imitar e chegar o mais próximo possível das propriedades que influenciam a administração de medicamentos, principalmente aquelas relacionadas a via oral. Desta forma, a previsão in silico indicou que as estruturas químicas 13 e 14 são possíveis candidatos a fármacos úteis para futuras triagens em ensaios realizados em células e em animais. As 15 moléculas inicialmente reprovadas nessa triagem podem sofrer modificações moleculares e triadas novamente com o intuito de melhorar suas propriedades farmacocinéticas e farmacodinâmicas.

A modelagem molecular executada nesse trabalho se mostrou uma abordagem fundamental na caracterização e na avaliação ADME-TOX de novos candidatos a fármacos contra o vírus da dengue.

\section{Conclusão}

O presente trabalho relata o desenho, a caracterização físico-química, farmacocinética e toxicológica de 17 candidatos a fármacos utilizados contra o vírus da dengue. Os resultados aqui alcançados revelaram que dois compostos análogos do ácido betulínico foram os que mostram maior relevância para o seguimento de desenvolvimento de novos candidatos a fármacos. Pode-se ainda destacar que as outras 15 estruturas podem ser otimizadas e passar por novas avaliações em computador para verificação da melhora da biodisponibilidade oral e redução da sua toxicidade. Assim, pode-se concluir que os compostos $13 \mathrm{e}$ 14 podem ser futuramente ensaiadas em modelos in vitro e in vivo. No entanto, cabe ainda ressaltar que investigações mais detalhadas, tais como perfil farmacocinético in vivo, toxicidade, mecanismo (s), são fundamentais para avaliar o potencial dessas moléculas contra a dengue.

Pretende-se futuramente realizar a síntese química e o ensaio farmacológico dos compostos 13 e 14 em modelo animal adequado para testar a ação dos fármacos anti-dengue.

\section{Referências}

Beesetti, H., Khanna, N., \& Swaminathan, S. (2016). Investigational drugs in early development for treating dengue infection. Expert Opinion on Investigational Drugs, 25 (9), 1059-69. https://doi.org/10.1080/13543784.2016.1201063 
Brady, O. J., \& Hay, S. I. (2020). The global expansion of dengue: How aedes aegypti mosquitoes enabled the first pandemic arbovirus. Annual Review of Entomology, 65, 191-208. https://doi.org/10.1146/annurev-ento-011019-024918

Daina, A., Michielin, O., \& Zoete, V. (2017). SwissADME: A free web tool to evaluate pharmacokinetics, drug-likeness and medicinal chemistry friendliness of small molecules. Scientific Reports, 7. https://doi.org/10.1038/srep42717

Dighe, S. N., Ekwudu, O., Dua, K., Chellappan, D. K., Katavic, P. L., \& Collet, T. A. (2019). Recent update on anti-dengue drug discovery. European Journal of Medicinal Chemistry, 176, 431-455. https://doi.org/10.1016/j.ejmech.2019.05.010

Ferreira, L. L. G., \& Andricopulo, A. D. (2019). ADMET modeling approaches in drug discovery. Drug Discovery Today, 24(5) 1157-65. https://doi.org/10.1016/j.drudis.2019.03.015

Gil, A.C. (2010). Como elaborar projetos de pesquisa. 5. ed. Atlas. São Paulo. Brasil.

Guy, B., Noriega, F., Ochiai, R. L., L'azou, M., Delore, V., Skipetrova, A., Verdier, F., Coudeville, L., Savarino, S., \& Jackson, N. (2017). A recombinant live attenuated tetravalent vaccine for the prevention of dengue. Expert Review of Vaccines, 16(7), 671-683. https://doi.org/10.1080/14760584.2017.1335201

Guzman, M. G., Gubler, D. J., Izquierdo, A., Martinez, E., \& Halstead, S. B. (2016). Dengue infection. Nature Reviews Disease Primers, 2(1), 1-25. https://doi.org/10.1038/nrdp.2016.55

Hage-Melim, L. I. da S., Federico, L. B., de Oliveira, N. K. S., Francisco, V. C. C., Correia, L. C., de Lima, H. B., Gomes, S. Q., Barcelos, M. P., Francischini, I. A. G., \& da Silva, C. H. T. de P. (2020). Virtual screening, ADME/Tox predictions and the drug repurposing concept for future use of old drugs against the COVID-19. Life Sciences, 256, 117963. https://doi.org/10.1016/j.lfs.2020.117963

Halim, S. A., Khan, S., Khan, A., Wadood, A., Mabood, F., Hussain, J., \& Al-Harrasi, A. (2017). Targeting Dengue Virus NS-3 Helicase by Ligand based Pharmacophore Modeling and Structure based Virtual Screening. Frontiers in Chemistry, 5. https://doi.org/10.3389/fchem.2017.00088

Kirchmair, J., Göller, A. H., Lang, D., Kunze, J., Testa, B., Wilson, I. D., Glen, R. C., \& Schneider, G. (2015). Predicting drug metabolism: Experiment and/or computation? Nature Reviews Drug Discovery, 14 (6), 387-404.https://doi.org/10.1038/nrd4581

Lakatos, E. M.; Marconi, M.A (2011). Metodologia científica. Brasil.

Lipinski, C. A., Lombardo, F., Dominy, B. W., \& Feeney, P. J. (1997). Experimental and computational approaches to estimate solubility and permeability in drug discovery and development settings. Advanced Drug Delivery Reviews, 23 (1-3), 3-25. https://doi.org/10.1016/S0169-409X(96)00423-1

Madden, J. C., Enoch, S. J., Paini, A., \& Cronin, M. T. D. (2020). A Review of In Silico Tools as Alternatives to Animal Testing: Principles, Resources and Applications. Alternatives to laboratory animals : ATLA, 48(4), 146-72. https://doi.org/10.1177/0261192920965977

Murugesan, A., \& Manoharan, M. (2019). Dengue virus. In Emerging and Reemerging Viral Pathogens: Volume 1: Fundamental and Basic Virology Aspects of Human, Animal and Plant Pathogens. 1, 281-359. https://doi.org/10.1016/B978-0-12-819400-3.00016-8

N. Powers, C., \& N. Setzer, W. (2016). An In-Silico Investigation of Phytochemicals as Antiviral Agents Against Dengue Fever. Combinatorial Chemistry \& High Throughput Screening, 19(7), 516-536. https://doi.org/10.2174/1386207319666160506123715

Patrick, Í., Amorim, S., Ramos Pestana, E., José, S., \& Mendes, F. (2017). Predição do metabolismo do candidato a fármaco cinamaldeído: Uma abordagem in silico. Revista Ceuma Perspectivas, 30(1), 111-120. http://smartcyp.sund.ku.dk/.

Pollett, S., Melendrez, M. C., Maljkovic Berry, I., Duchêne, S., Salje, H., Cummings, D. A. T., \& Jarman, R. G. (2018). Understanding dengue virus evolution to support epidemic surveillance and counter-measure development. Infection, Genetics and Evolution, 62, 279-95. https://doi.org/10.1016/j.meegid.2018.04.032

Rai, J., \& Kaushik, K. (2018). Reduction of Animal Sacrifice in Biomedical Science \& Research through Alternative Design of Animal Experiments. Saudi Pharmaceutical Journal, 26(6), 896-902.https://doi.org/10.1016/j.jsps.2018.03.006

Rao, V. S., \& Srinivas, K. (2011). Modern drug discovery process: An in silico approach. Journal of Bioinformatics and Sequence Analysis, 2(5), 89-94. http://www.academicjournals.org/JBSA

Thangarasu, P., Thamarai Selvi, S., \& Manikandan, A. (2018). Unveiling novel 2-cyclopropyl-3-ethynyl-4-(4-fluorophenyl)quinolines as GPCR ligands via PI3-kinase/PAR-1 antagonism and platelet aggregation valuations; development of a new class of anticancer drugs with thrombolytic effects. Bioorganic Chemistry, 81, 468-480. https://doi.org/10.1016/j.bioorg.2018.09.011

Vavougios, G. D., Zarogiannis, S. G., Krogfelt, K. A., Gourgoulianis, K., Mitsikostas, D. D., \& Hadjigeorgiou, G. (2018). Novel candidate genes of the PARK7 interactome as mediators of apoptosis and acetylation in multiple sclerosis: An in silico analysis. Multiple Sclerosis and Related Disorders, $19,8-14$. https://doi.org/10.1016/j.msard.2017.10.013

Vera, A. A. (1989). Metodologia da pesquisa científica. (8a ed.), Brasil. 
Research, Society and Development, v. 10, n. 4, e53010414459, 2021

(CC BY 4.0) | ISSN 2525-3409 | DOI: http://dx.doi.org/10.33448/rsd-v10i4.14459

Vergara, S. C. (2006) Projetos e relatórios de pesquisa em administração. (5a ed.), Brasil.

Vukic, V. R., Loncar, D. M., Vukic, D. V., Jevric, L. R., Benedekovic, G., Francuz, J., Kojic, V., Karadzic Banjac, M. Z., \& Popsavin, V. (2019). In vitro antitumor activity, ADME-Tox and 3D-QSAR of synthesized and selected natural styryl lactones. Computational Biology and Chemistry, 83, 107112. https://doi.org/10.1016/j.compbiolchem.2019.107112

Waman, V. P., Kolekar, P., Ramtirthkar, M. R., Kale, M. M., \& Kulkarni-Kale, U. (2016). Analysis of genotype diversity and evolution of Dengue virus serotype 2 using complete genomes. PeerJ, 8. https://doi.org/10.7717/peerj.2326 